\title{
Modifying an approximation process using non-Newtonian calculus
}

\author{
Octavian Agratini and Harun Karsli
}

\begin{abstract}
In the present note we modify a linear positive Markov process of discrete type by using so called multiplicative calculus. In this framework, a convergence property and the error of approximation are established. In the final part some numerical examples are delivered.
\end{abstract}

Mathematics Subject Classification (2010): 41A36, 47S30.

Keywords: Linear positive operator, non-Newtonian calculus, modulus of multiplicative smoothness.

\section{Introduction}

The study of the linear methods of approximation, which are given by sequences of linear and positive operators, has become a firmly rooted part of an old area of mathematical research called Approximation Theory. It has a great potential for applications to a wide variety of issues.

The starting point of this note is a general approximation process of discrete type which acts on the real valued functions defined on a compact interval $K \subset \mathbb{R}$. Since a linear substitution maps any compact interval $[a, b]$ into $[0,1]$, we will only consider functions defined on $[0,1]$. Each operator $L_{n}$ of the class to which we refer, uses an equidistant network with a flexible step of the form $\Delta_{n}=\left(k \lambda_{n}\right)_{0 \leq k \leq n}$, where $\left(\lambda_{n}\right)_{n \geq 1}$ is a strictly decreasing sequence of real numbers with the property

$$
0<\lambda_{n} \leq \frac{1}{n}, n \in \mathbb{N}
$$

The operators we are referring to are designed as follows

$$
\left(L_{n} f\right)(x)=\sum_{k=0}^{n} a_{k}\left(\lambda_{n} ; x\right) f\left(k \lambda_{n}\right), n \in \mathbb{N}, x \in[0,1],
$$


where the function $a_{k}\left(\lambda_{n} ; \cdot\right):[0,1] \rightarrow \mathbb{R}_{+}$is continuous for each $n \in \mathbb{N}$ and $k \in\{0,1, \ldots, n\}$. The condition (1.2) guarantees that $\Delta_{n}$ is indeed a network on the compact $[0,1]$.

Typically, the operators described by (1.2) satisfy the condition of reproducing constants. Being linear operators, this property is involved in achieving the following identity

$$
\sum_{k=0}^{n} a_{k}\left(\lambda_{n} ; x\right)=1, x \in[0,1] .
$$

Throughout the paper, we consider this as a working hypothesis. Note that such operators are called Markov operators.

At this point we refer to non-Newtonian calculus also called as multiplicative calculus. In the 1970s, Michael Grossman and Robert Katz [5] have developed this type of calculation moving the roles of subtraction and addition to division and multiplication. See also Dick Stanley's paper [8]. This type of calculus was also called geometric calculus in order to emphasize that changes in function arguments are measured by differences, while changes in values are measured in ratios. Recently, Bashirov et al. [1] have given the complete mathematical description of multiplicative calculus. In the last decade there have been extensions of this notion in different directions of mathematics, even if it has a relatively restrictive area of applications than the classical calculus of Newton and Leibniz covering only positive quantities. Of the area where this type of approach has proven efficacy, we mention the theory of economic growth, see, for example, [4].

In the present paper our aim is to bring up multiplicative calculus to the attention of researchers in the branch of positive approximation processes. We could find no references that treat any kind of multiplicative calculus to the above mentioned directions.

In the next two sections we present basic elements of multiplicative calculus and new results as regards the positive approximation processes. A particular case is treated at the end of the paper.

\section{Preliminaries}

We introduce the second central moment of $L_{n}, n \in \mathbb{N}$, operators, i.e.,

$$
\mathcal{M}_{2}\left(L_{n} ; x\right):=\left(L_{n} \varphi_{x}^{2}\right)(x) \text {, where } \varphi_{x}(t)=|t-x|,
$$

$(t, x) \in[0,1] \times[0,1]$. Taking into account Bohman-Korovkin criterion, since (1.3) is fulfilled, in order the sequence $\left(L_{n}\right)_{n \geq 1}$ to become an approximation process on the space $C([0,1])$, it is necessary and sufficient to take place the following relation

$$
\lim _{n \rightarrow \infty} \mathcal{M}_{2}\left(L_{n} ; x\right)=0, \quad \text { uniformly in } x \in[0,1] .
$$

We also consider that this identity is achieved.

Set $\mathbb{R}_{+}^{*}=(0, \infty)$. Also, $B^{+}([0,1])$ stands for all strictly positive real valued functions defined on $[0,1]$ and

$$
C^{+}([0,1])=\left\{f \in B^{+}([0,1]): f \text { continuous on }[0,1]\right\} \text {. }
$$


We collected some information about multiplicative calculus. Here, the symbol $\ominus$ represents the difference in non-Newtonian geometric calculus which means the division in the classical calculus. Consequently, $a \ominus b$ means $a / b$, provided that $a / b$ makes sense.

In non-Newtonian geometric calculus, the multiplicative absolute value (or modulus) of an element $x \in(0, \infty)$ be a number $|x|^{*}$ such that

$$
|x|^{*}= \begin{cases}x & , \quad x>1 \\ 1 & , \quad x=1 \\ 1 / x & , \quad x<1\end{cases}
$$

Owing to the definition of multiplicative absolute value, the multiplicative distance between two elements $x, y \in(0, \infty)$ is given by

$$
|x \ominus y|^{*}=\left|\frac{x}{y}\right|^{*}= \begin{cases}x / y & , \quad x / y>1 \\ 1 & , \quad x=y \\ y / x & , \quad x / y<1\end{cases}
$$

From the definition of the multiplicative absolute value, it is obvious that $|x|^{*} \geq 1$ for all $x \in(0, \infty)$. following

For a closed interval $I \subseteq \mathbb{R}$, denoting $\left\{f \mid f: I \rightarrow \mathbb{R}_{+}^{*}\right\}=\mathcal{F}^{+}(I)$, we present the

Definition 2.1. A function $f \in \mathcal{F}^{+}(I)$ is said to tend to the limit $L>0$ as $x$ tends to $a \in I$, if, corresponding to any arbitrary chosen number $\varepsilon>1$, there exists a positive number $\delta>1$ such that

$$
|f(x) \ominus L|^{*}<\varepsilon
$$

for all values of $x$ for which

$$
1<|x \ominus a|^{*}<\delta
$$

Here

and

$$
1<|x \ominus a|^{*}<\delta \Longleftrightarrow \frac{a}{\delta}<x<a \delta
$$

$$
|f(x) \ominus L|^{*}<\varepsilon \Longleftrightarrow \frac{L}{\varepsilon} \leq f(x)<L \varepsilon .
$$

We use the notation $\lim _{x \rightarrow a} f(x) \stackrel{m}{=} L$ or $f(x) \stackrel{m}{\longrightarrow} L, x \rightarrow a$.

Definition 2.2. A function $f \in \mathcal{F}^{+}(I)$ is said to be multiplicative continuous at $x=a \in I$, if

holds.

$$
\lim _{x \rightarrow a} f(x) \stackrel{m}{=} f(a)
$$

In other words, a function $f \in \mathcal{F}^{+}(I)$ is said to be multiplicative continuous at $x=a \in I$, if, corresponding to any arbitrary chosen number $\varepsilon>1$, there exists a positive number $\delta>1$ such that

$$
|f(x) \ominus f(a)|^{*}<\varepsilon
$$

for all values of $x$ for which

$$
|x \ominus a|^{*}<\delta .
$$


Similar to the classic modulus of smoothness, can be defined the modulus of multiplicative smoothness.

Definition 2.3. Let $I \subseteq \mathbb{R}_{+}$be an interval and $f \in B^{+}(I)$. The modulus of multiplicative smoothness of $f$ is denoted by $\omega^{\langle m\rangle}(f ; \cdot)$ and is defined as follows

$$
\omega^{\langle m\rangle}(f ; \delta)=\sup _{\substack{1 \leq|x \ominus t| \leq \delta \\ x, t \in I}}|f(x) \ominus f(t)|^{*}, \delta \geq 1 .
$$

Remark 2.4. Examining relation (2.2) we deduce

i) $\omega^{\langle m\rangle}(f ; 1)=1$;

ii) if $1 \leq \delta_{1}<\delta_{2}$, then $\omega^{\langle m\rangle}\left(f ; \delta_{1}\right) \leq \omega^{\langle m\rangle}\left(f ; \delta_{2}\right)$, consequently $\omega^{\langle m\rangle}(f ; \cdot)$ is a non-decreasing function.

We associate to the operators defined by (1.2) with the fulfillment of hypotheses (1.3) and (2.1), the following operators

$$
\left(L_{n}^{\langle m\rangle} f\right)(x)=\prod_{k=0}^{n}\left(f\left(k \lambda_{n}\right)\right)^{a_{k}\left(\lambda_{n} ; x\right)}, x \in[0,1],
$$

for each function $f \in B^{+}([0,1])$. This new class of operators loses the linearity property.

We also notice that it keeps the constants. Indeed, by virtue of property (1.3), if $f(x)=c>0, x \in[0,1]$, then $\left(L_{n}^{\langle m\rangle} c\right)(x)=c, x \in[0,1]$.

Further on, our goal is to highlight approximation properties of the sequence $\left(L_{n}^{\langle m\rangle}\right)_{n \geq 1}$.

\section{Results}

Theorem 3.1. Let $f \in B^{+}([0,1])$ and the operators $L_{n}^{\langle m\rangle}, n \in \mathbb{N}$, be defined by (2.3). The following relation

$$
\lim _{n \rightarrow \infty}\left(L_{n}^{\langle m\rangle} f\right)\left(x_{0}\right) \stackrel{m}{=} f\left(x_{0}\right)
$$

holds at each point $x_{0} \in(0,1]$ of multiplicative continuity of $f$.

Proof. Let $\varepsilon>1$ be arbitrarily fixed. In order to prove the theorem we have to show

$$
\left|\left(L_{n}^{\langle m\rangle} f\right)\left(x_{0}\right) \ominus f\left(x_{0}\right)\right|^{*}<\varepsilon
$$

holds true at each point $x_{0} \in(0,1]$ of multiplicative continuity of $f \in B^{+}([0,1])$.

If $f \in B^{+}([0,1])$ is a constant function then one has

$$
\left(L_{n}^{\langle m\rangle} f\right)\left(x_{0}\right)=f\left(x_{0}\right),
$$

and hence

$$
\left|\left(L_{n}^{\langle m\rangle} f\right)\left(x_{0}\right) \ominus f\left(x_{0}\right)\right|^{*}=1<\varepsilon
$$

holds true at every point $x_{0} \in(0,1]$. This proves $(3.1)$ for constant functions. 
Now, we assume that $f \in B^{+}([0,1])$ is not a constant function. Since the multiplicative absolute value is always greater than or equal to $1\left(|\bullet|^{*} \geq 1\right)$, it is sufficient only to show that the inequality

$$
\left|\left(L_{n}^{\langle m\rangle} f\right)\left(x_{0}\right) \ominus f\left(x_{0}\right)\right|^{*}<\varepsilon
$$

is valid for $n \geq N, N$ being a certain rank. By using (2.3) and (1.3) we can write

$$
\left|\left(L_{n}^{\langle m\rangle} f\right)\left(x_{0}\right) \ominus f\left(x_{0}\right)\right|^{*}=\left|\prod_{k=0}^{n}\left(\frac{f\left(k \lambda_{n}\right)}{f\left(x_{0}\right)}\right)^{a_{k}\left(\lambda_{n} ; x_{0}\right)}\right|^{*} .
$$

Since $\lim _{x \rightarrow x_{0}} f(x) \stackrel{m}{=} f\left(x_{0}\right)$, in accordance with Definition 2.2 , there exists a positive number $\delta>1$ such that

$$
\left|f(x) \ominus f\left(x_{0}\right)\right|^{*}<\varepsilon
$$

for all values of $x$ for which

$$
\left|x \ominus x_{0}\right|^{*}<\delta .
$$

We split up the set $J=\{0,1, \ldots, n\}$ as follows

$$
\begin{aligned}
& J_{0}=\{0\}, \\
& J_{1}=\left\{k \in J \backslash J_{0}:\left|k \lambda_{n} \ominus x_{0}\right|^{*}<\delta\right\}, \\
& J_{2}=\left\{k \in J \backslash J_{0}:\left|k \lambda_{n} \ominus x_{0}\right|^{*} \geq \delta\right\} .
\end{aligned}
$$

Returning at (3.2) we break down the product as follows

$$
\begin{aligned}
& \left|\prod_{k=0}^{n}\left(\frac{f\left(k \lambda_{n}\right)}{f\left(x_{0}\right)}\right)^{a_{k}\left(\lambda_{n} ; x_{0}\right)}\right|^{*} \\
\leq & \left(\left|\frac{f(0)}{f\left(x_{0}\right)}\right|^{*}\right)^{a_{0}\left(\lambda_{n} ; x_{0}\right)} \prod_{k \in J_{1}}\left(\left|\frac{f\left(k \lambda_{n}\right)}{f\left(x_{0}\right)}\right|^{*}\right)^{a_{k}\left(\lambda_{n} ; x_{0}\right)} \prod_{k \in J_{2}}\left(\left|\frac{f\left(k \lambda_{n}\right)}{f\left(x_{0}\right)}\right|^{*}\right)^{a_{k}\left(\lambda_{n} ; x_{0}\right)} .
\end{aligned}
$$

The first product can be increased in the following way

$$
\prod_{k \in J_{1}}\left(\left|\frac{f\left(k \lambda_{n}\right)}{f\left(x_{0}\right)}\right|^{*}\right)^{a_{k}\left(\lambda_{n} ; x_{0}\right)}<\varepsilon^{\sum_{k \in J_{1}} a_{k}\left(\lambda_{n} ; x_{0}\right)} \leq \varepsilon,
$$

see (3.3) and (1.3). The relation $k \in J_{0} \cup J_{2}$ involves

$$
\begin{aligned}
\sum_{k \in J_{0} \cup J_{2}} a_{k}\left(\lambda_{n} ; x_{0}\right) & \leq \sum_{k \in J_{0} \cup J_{2}} \frac{\left(k \lambda_{n}-x_{0}\right)^{2}}{\delta^{2}} a_{k}\left(\lambda_{n} ; x_{0}\right) \\
& \leq \frac{1}{\delta^{2}} \sum_{k=0}^{n}\left(k \lambda_{n}-x_{0}\right)^{2} a_{k}\left(\lambda_{n} ; x_{0}\right) \\
& =\frac{1}{\delta^{2}} \mathcal{M}_{2}\left(L_{n} ; x_{0}\right) .
\end{aligned}
$$

Based on (2.1), for any $\mu>0$, there exists a rank $N \in \mathbb{N}$ such that

$$
\mathcal{M}_{2}\left(L_{n} ; x_{0}\right)<\mu \text {, for every } n \geq N \text {. }
$$


Setting

$$
\sup _{x \in[0,1]}\left|\frac{f(x)}{f\left(x_{0}\right)}\right|^{*}=M
$$

we can evaluate the last part from (3.6)

$$
\prod_{k \in J_{0} \cup J_{2}}\left(\left|\frac{f\left(k \lambda_{n}\right)}{f\left(x_{0}\right)}\right|^{*}\right)^{a_{k}\left(\lambda_{n} ; x_{0}\right)} \leq M^{\sum_{k \in J_{0} \cup J_{2}}\left(\lambda_{n} ; x_{0}\right)} \leq M^{\mu / \delta^{2}}, n \geq N,
$$

see (3.7) and (3.8). Returning at (3.6), we get

$$
\forall \mu>0, \exists N \in \mathbb{N}, \forall n \geq N, \prod_{k=0}^{n}\left|\frac{f\left(k \lambda_{n}\right)}{f\left(x_{0}\right)}\right|^{a_{k}\left(\lambda_{n} ; x_{0}\right)}<\varepsilon M^{\mu / \delta^{2}} .
$$

If $M=1$, then we obtain exactly the inequality (3.2). Otherwise $(M>1)$, choosing $\mu=\delta^{2} \log _{M} \varepsilon>0$, we obtain the same inequality from (3.2) with $\varepsilon:=\varepsilon^{2}$ which does not alter the statement.

In this moment, the proof of (3.2) is completed, consequently (3.1) takes place.

Next, we establish an upper bound of the error of approximation by using the modulus of multiplicative smoothness.

Theorem 3.2. Let $f \in B^{+}([0,1])$ and the operators $L_{n}^{\langle m\rangle}, n \in \mathbb{N}$, be defined by (2.3). For $n$ large enough, the following relation

$$
\left|\left(L_{n}^{\langle m\rangle} f\right)(x) \ominus f\left(x_{0}\right)\right|^{*} \leq M \omega^{\langle m\rangle}(f ; \delta), \delta \geq 1,
$$

holds at each point $x_{0} \in(0,1]$ of multiplicative continuity of $f$. The constant $M$ is defined at (3.9).

Proof. We use the identity (3.3) and the decomposition of that product according to the relation (3.6). Based both on the definition of $\omega^{\langle m\rangle}(f ; \cdot)$, see $(2.2)$, and the inequalities set out in (3.10) that are valid for any $\mu>0$ and $n$ sufficiently large, we can write

$$
\begin{aligned}
\left|\left(L_{n}^{\langle m\rangle} f\right)\left(x_{0}\right) \ominus f\left(x_{0}\right)\right|^{*} & \leq\left(\prod_{\left|k \lambda_{n} \ominus x_{0}\right|^{*} \leq \delta} \omega^{\langle m\rangle}(f ; \delta)^{a_{k}\left(\lambda_{n} ; x_{0}\right)}\right) M^{\mu / \delta^{2}} \\
& \leq M^{\mu / \delta^{2}} \omega^{\langle m\rangle}(f ; \delta) .
\end{aligned}
$$

Choosing $\mu=\delta^{2}$, we obtain the desired result.

\section{A special case}

In this section, we give a particular example of operators satisfying the assumptions employed in the previous sections. 


\subsection{Multiplicative (geometric) Bernstein operators}

By choosing $\lambda_{n}=\frac{1}{n}, a_{k}\left(\lambda_{n} ; x\right)=p_{n, k}(x)$, where $p_{n, k}(x)=\left(\begin{array}{c}n \\ k\end{array}\right) x^{k}(1-x)^{n-k}$ is the Bernstein basis, then we obtain a special case of the operators (2.3), namely multiplicative (geometric) version of the celebrated Bernstein operators. More precisely, $B_{n}^{\langle m\rangle}: B^{+}[0,1] \rightarrow C^{+}[0,1](n \geq 1)$ is given by

$$
\left(B_{n}^{\langle m\rangle} f\right)(x)=\prod_{k=0}^{n}\left[f\left(\frac{k}{n}\right)\right]^{p_{n, k}(x)}, x \in[0,1] .
$$

As a consequence of Theorem 3.1 for functions $f \in B^{+}([0,1])$ and for multiplicative (geometric) Bernstein operators $B_{n}^{\langle m\rangle}$, we have the following direct estimate:

Corollary 4.1. Let $f \in B^{+}([0,1])$ be a function. Let the operators $B_{n}^{\langle m\rangle}, n \in \mathbb{N}$, be defined by (4.1). The following relation

$$
\lim _{n \rightarrow \infty}\left(B_{n}^{\langle m\rangle} f\right)\left(x_{0}\right) \stackrel{m}{=} f\left(x_{0}\right)
$$

holds at each point $x_{0} \in(0,1]$ of multiplicative continuity of $f$.

\subsection{Graphical and Numerical Representations}

In the recent period, many operators have been investigated that generalize the classical approximation operators and the theoretical approach is usually accompanied by illustrations of convergence properties of particular functions. The included graphics are realized using software programs. Among such papers, we randomly quote [3], [7], [2], [6], the last three appeared in the years 2019-2020.

Following this line, we give some graphical and numerical examples to illustrate the approximation results for multiplicative (geometric) Bernstein operators obtained in the present paper.

We note that in the Figures 1,2 and 3, the graph with the red line belongs to the original function, the graph with the green line to the operators with $n=2$, and finally the graph consisting of blue line to the operators with $n=10$.

Example 4.2. Let us consider the function $f(x)=x^{3}+1$, and we take its corresponding multiplicative Bernstein operator $\left(B_{n}^{\langle m\rangle} f\right)(x)$ (4.1), that one has for $n=2$ and for $n=10$. 


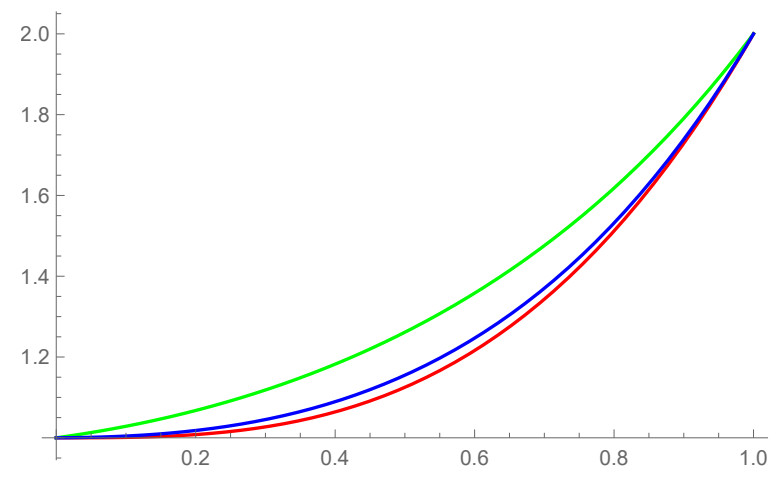

Figure 1. Approximation of $f(x)=x^{3}+1$ by multiplicative Bernstein operators (4.1), for $n=2$ and $n=10$.

In the following tables, by using the Wolfram Mathematica 11 Program, we compute the error of approximation numerically at certain points for $n=100,300$ and 500;

\begin{tabular}{|cccc|}
\hline$\underline{x=0.2}$ & $\underline{n=100}$ & $\underline{n=300}$ & $\underline{n=500}$ \\
$\left(B_{n}^{\langle m\rangle} f\right)(0.2)$ & 1.00896 & 1.00832 & 1.00819 \\
$f(0.2)$ & 1.008 & 1.008 & 1.008 \\
The Error & 0.000955622 & 0.000316981 & 0.000189999 \\
\hline
\end{tabular}

\begin{tabular}{|cccc|}
\hline$\frac{x=0.5}{\left\langle\left(B_{n}^{\langle m\rangle} f\right)(0.5)\right.}$ & $\underline{n=100}$ & $\underline{n=300}$ & $\underline{n=500}$ \\
$f(0.5)$ & 1.12811 & 1.12604 & 1.12562 \\
The Error & 0.00310983 & 0.00103996 & 0.000624384 \\
\hline
\end{tabular}

and finally

\begin{tabular}{|cccc|}
\hline$\left.\frac{x=0.8}{\langle m\rangle} f\right)(0.8)$ & $\underline{n=100}$ & $\underline{n=300}$ & $\underline{n=500}$ \\
$f(0.8)$ & 1.5139 & 1.51263 & 1.51238 \\
The Error & 0.00189809 & 0.000630805 & 0.000378252 \\
\hline
\end{tabular}

Example 4.3. Let us consider the function $f(x)=\sin (x+1)$, and we take its corresponding multiplicative Bernstein operator $\left(B_{n}^{\langle m\rangle} f\right)(x)(4.1)$, that one has for $n=2$ and for $n=10$. 


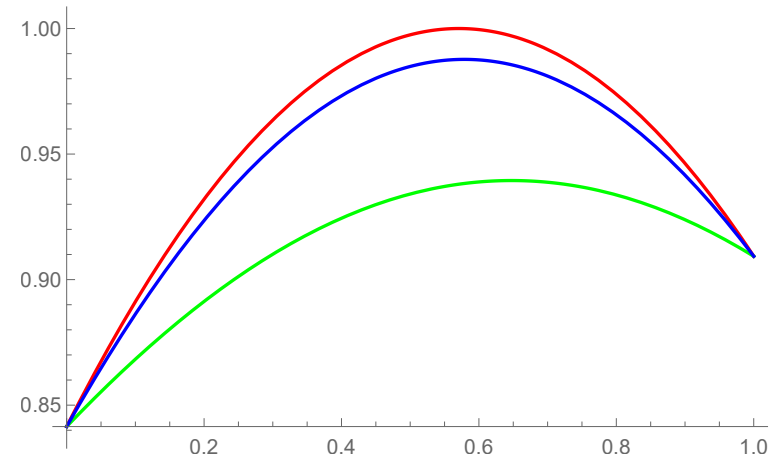

Figure 2. Approximation of $f(x)=\sin (x+1)$ by multiplicative Bernstein operators (4.1), for $n=2$ and $n=10$.

In the following tables, we compute the error of approximation numerically at certain points for $n=100,300$ and 500 ;

\begin{tabular}{|cccc|}
\hline$\underline{x=0.2}$ & $\underline{n=100}$ & $\underline{n=300}$ & $\underline{n=500}$ \\
$\left(B_{n}^{\langle m\rangle} f\right)(0.2)$ & 0.931181 & 0.931753 & 0.931867 \\
$f(0.2)$ & 0.932039 & 0.932039 & 0.932039 \\
The Error & 0.000857595 & 0.000286029 & 0.000171637 \\
\hline
\end{tabular}

\begin{tabular}{|cccc|}
\hline$\underline{x=0.5}$ & $\underline{n=100}$ & $\underline{n=300}$ & $\underline{n=500}$ \\
$\left(B_{n}^{\langle m\rangle} f\right)(0.5)$ & 0.996241 & 0.997077 & 0.997244 \\
$f(0.5)$ & 0.997495 & 0.997495 & 0.997495 \\
The Error & 0.00125394 & 0.000417802 & 0.00025066 \\
\hline
\end{tabular}

and finally

\begin{tabular}{|cccc|}
\hline$\underline{x=0.8}$ & $\underline{n=100}$ & $\underline{n=300}$ & $\underline{n=500}$ \\
$\left(B_{n}^{\langle m\rangle} f\right)(0.8)$ & 0.973026 & 0.973574 & 0.973683 \\
$f(0.8)$ & 0.973848 & 0.973848 & 0.973848 \\
The Error & 0.000821133 & 0.000273789 & 0.000164283 \\
\hline
\end{tabular}

Example 4.4. Let us consider the function $f(x)=\sin x+1$, and we take its corresponding multiplicative Bernstein operator $\left(B_{n}^{\langle m\rangle} f\right)(x)(4.1)$, that one has for $n=2$ and for $n=10$. 


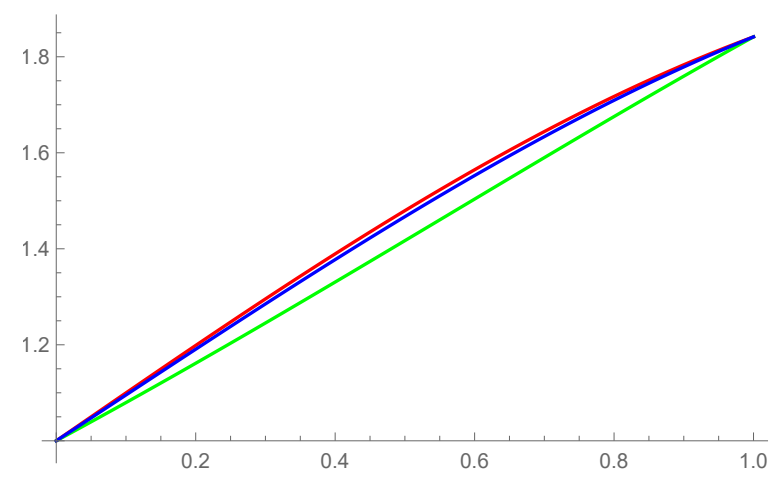

Figure 3. Approximation of $f(x)=\sin x+1$ by multiplicative Bernstein operators (4.1), for $n=2$ and $n=10$.

In the following tables, we compute the error of approximation numerically at certain points for $n=100,300$ and 500 ;

\begin{tabular}{|cccc|}
\hline$\left.\frac{x=0.2}{\langle m\rangle} f\right)(0.2)$ & 1.19787 & $\underline{n=300}$ & $\underline{n=500}$ \\
$\left(B_{n}^{\langle m}(0.2)\right.$ & 1.19867 & 1.19867 & 1.19851 \\
The Error & 0.000798903 & 0.000266545 & 0.000159956 \\
\hline
\end{tabular}

\begin{tabular}{|cccc|}
\hline$\frac{x=0.5}{\left\langle B_{n}^{\langle m\rangle} f\right)(0.5)}$ & $\underline{n=100}$ & $\underline{n=300}$ & $\underline{n=500}$ \\
$f(0.5)$ & 1.47918 & 1.47901 & 1.47918 \\
The Error & 0.00125027 & 0.000416697 & 0.000250011 \\
\hline
\end{tabular}

and finally

\begin{tabular}{|cccc|}
\hline$\underline{x=0.8}$ & $\underline{n=100}$ & $\underline{n=300}$ & $\underline{n=500}$ \\
$\left(B_{n}^{\langle m\rangle} f\right)(0.8)$ & 1.71656 & 1.71709 & 1.7172 \\
$f(0.8)$ & 1.71736 & 1.71736 & 1.71736 \\
The Error & 0.000800703 & 0.000266745 & 0.000160028 \\
\hline
\end{tabular}

Remark 4.5. Unlike error evaluation for linear and positive operators, from (3.11) we cannot deduce the convergence property of the sequence $\left(L_{n}^{\langle m\rangle} f\right)_{n \geq 1}$ to $f$. This note should be regarded as a pioneering activity in order to introduce multiplicative calculus in the field promoted by Korovkin type theory.

\section{References}

[1] Bashirov, A.E., Kurpinar, E.M., Özyapici, A., Multiplicative calculus and its applications, J. Math. Anal. Appl., 337(2008), 36-48.

[2] Cai, Q.-Bo, Zhou, G., Li, J., Statistical approximation properties of $\lambda$-Bernstein operators based on q-integers, Open Math., 17(2019), no. 1, 487-498. 
[3] Cătinaş, T., Some classes of surfaces generated by Nielson and Marshall type operators on the triangle with one curved side, Stud. Univ. Babeş-Bolyai Math., 61(2016), no. 3, 305-314.

[4] Filip, D.A., Piatecki, C., A non-newtonian examination of the theory of exogenous economic growth, Mathematica Aeterna, 4(2014), no. 2, 101-117.

[5] Grossman, M., Katz, R., Non-Newtonian Calculus, Lee Press, Pigeon Cove, MA, 1972.

[6] Gupta, V., Agrawal, G., Approximation for link Ismail-May operators, Ann. Funct. Anal., https://doi.org/10.1007/s43034-019-00051-y, online: 13 January 2020.

[7] Mursaleen, M., Rahman, S., Ansari, K.J., Approximation by Jakimovski-LeviatanStancu-Durrmeyer type operators, Filomat, 33(2019), no. 6, 1517-1530.

[8] Stanley, D., A multiplicative calculus, Primus: Problems, Resources, and Issues in Mathematics Undergraduate Studies, 9(1999), no. 4, 310-326.

Octavian Agratini

Babeş-Bolyai University, Faculty of Mathematics and Computer Science

Str. Kogălniceanu, 1, 400084 Cluj-Napoca, Romania

and

Tiberiu Popoviciu Institute of Numerical Analysis, Romanian Academy

Str. Fântânele, 57, 400320 Cluj-Napoca, Romania

e-mail: agratini@math.ubbcluj.ro

Harun Karsli

Bolu Abant Izzet Baysal University Faculty of Science and Arts

Department of Mathematics

14030 Golkoy Bolu, Turkey

e-mail: karsli_h@ibu.edu.tr 